\title{
Flow Cytometry and cell sorting: pushing the technology to the limit
}

\author{
Ger van den Engh
}

Institute for Systems Biology, 1441 North $34^{\text {th }}$ Street, Seattle, WA 98103

Cytopeia, Inc., $1273028^{\text {th }}$ Avenue NE, Seattle, WA 98125

Flow cytometry and cell sorting have proven to be valuable tools in experimental cell biology, especially immunology. The hemopoetic tissues contain an extremely heterogeneous population of identical looking cells. The differences in cell function are not manifested in cell morphology but can be detected by the expression density of various surface proteins. By generating membrane-antigen specific antibodies coupled to fluorescent dyes it has become possible to explore the diversity among lymphocytes. The ability to use quantitative fluorescence signals to viably separate cells by cell sorting allows one to link the observed heterogeneity to cell function. Quantitative fluorescence measurements in combination with cell sorting offer unique insights into the cellular interactions that place during the immune response.

The above example illustrates the requirements for a useful sorting technology. To see the differences between the various cell types the fluorescence analysis must be as accurate as possible and must extend over a large dynamic range. Because of the extreme functional heterogeneity and the fact that small groups of cells may rapidly expand by proliferation, many of the pivotal cell types occur at a low incidence. Functional studies (manipulation in vitro) often require significant numbers of cells. For instance, fewer than 1 in a thousand cells in the bone marrow is a stem cell (by themselves considered a heterogeneous population). To repopulate a mouse about a thousand purified stems cells are needed. An experiment with the proper experimental and control groups easily requires the inspection and sorting of tens of millions of cells. At a thousand cells a second such an experiment may take a few hours; a lot longer if larger mammals than mice are used.

In developing sorting technology it is important that the instruments are as fast as possible without compromising measurement precision and sensitivity. The highperformance instruments must also be practical to use. If we are successful, sorting experiments will lead to medical applications and the instruments must meet extreme requirements of reliability and safety.

This presentation will explain the principles of flow cytometry and cell sorting and consider how the underlying physics affects instrument performance. Factors such as polarization, energy transfer, spectral analysis and photon statistics that are often ignored but are critical the final precision. Examples of the optics and electronics of highperformance cell sorter designs will be presented. Finally an illustration of the use of advanced sorting techniques in special biological problems and shows how the study of non-linear fluorescence phenomena can help reveal details of dye binding and cell function. 


\section{a flow cytometer is a parfocal microscope}

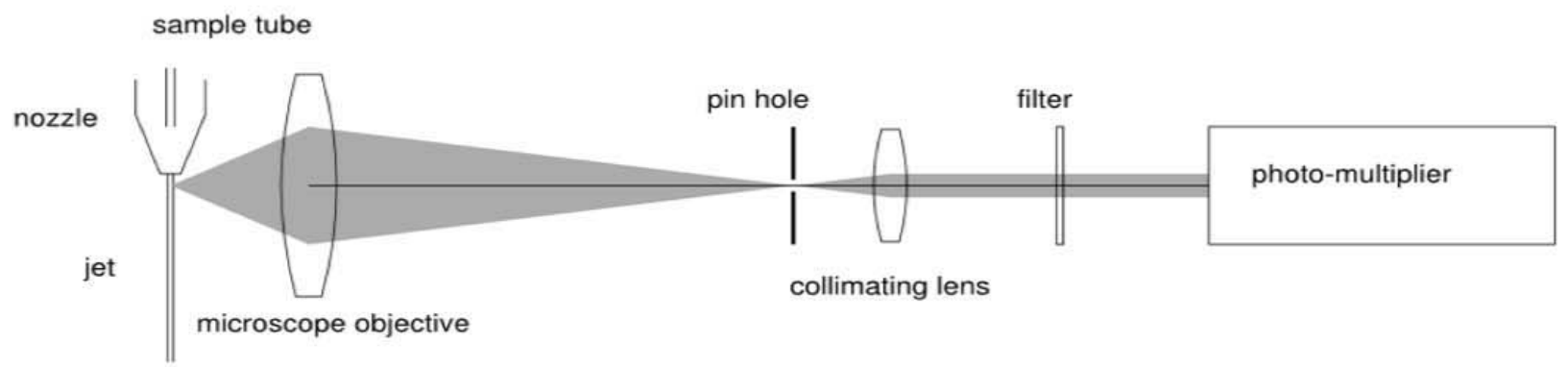

How cell sorting works

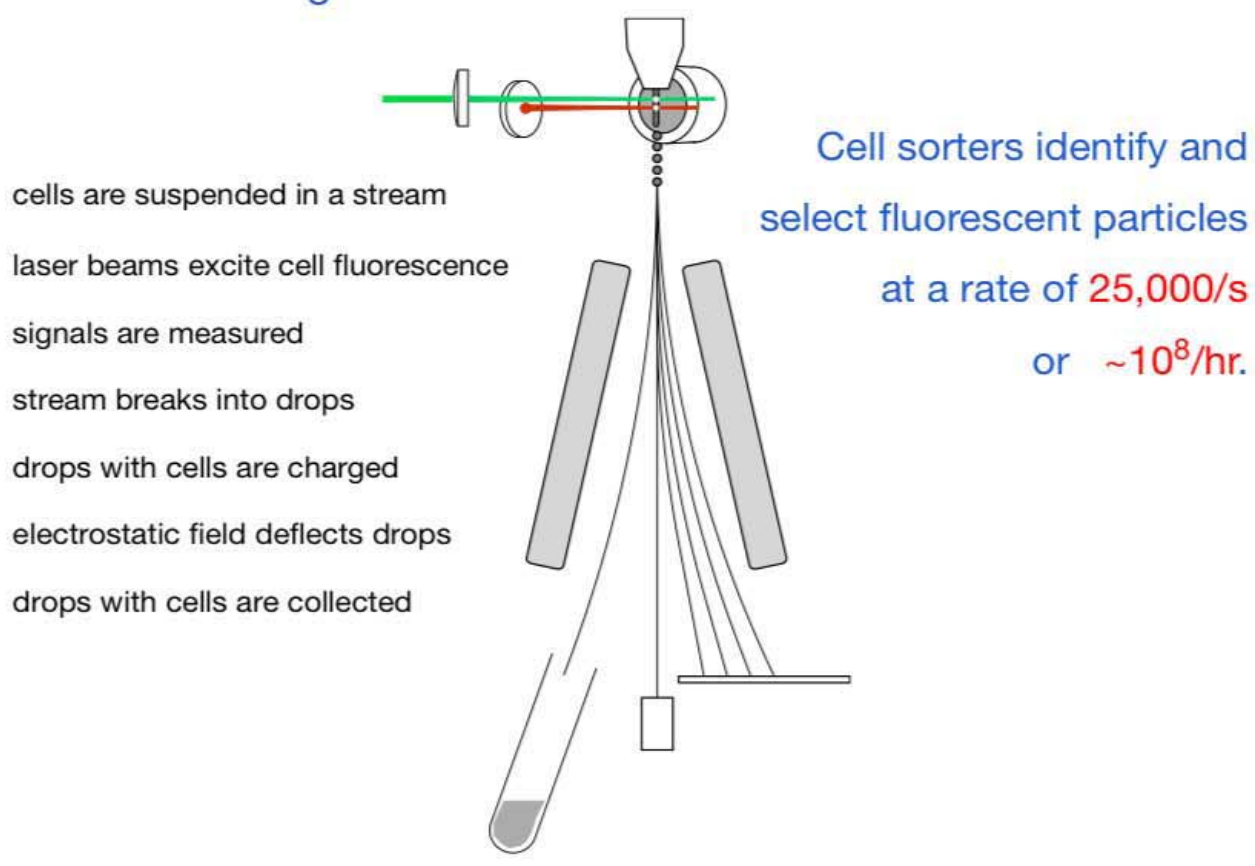

References

Shapiro H, (2001) Practical Flow Cytometry. Wiley, NewYork.

van den Engh, G. (2000) High-speed cell sorting. in: Robinson, P., and Durack, J. (eds.) Single cell Sorting Techniques. Wiley, New York.

Asbury, C., Uy, J., van den Engh, G. (2000) Polarization of fluorescence and scatter signals in flow cytometry. Cytometry 40:88-101.

Petersen TW and van den Engh G. (2003) Stability of the Break-off Point in a High-Speed Cell Sorter. Cytometry 56A:63-70.

Ibrahim, SF, van den Engh, G. (2004) High-speed chromosome sorting. Chromosome Research 12:5-14. 